\title{
A study on user preference of high dynamic range over low dynamic range video
}

\author{
Ratnajit Mukherjee $^{1}$ - Kurt Debattista ${ }^{1}$ - Thomas Bashford-Rogers ${ }^{1}$. \\ Brian Waterfield $^{2}$. Alan Chalmers ${ }^{1}$
}

Published online: 13 May 2016

(C) The Author(s) 2016. This article is published with open access at Springerlink.com

\begin{abstract}
The increased interest in high dynamic range (HDR) video over existing low dynamic range (LDR) video during the past decade or so was primarily due to its inherent capability to capture, store and display the full range of real-world lighting visible to the human eye with increased precision. This has led to an inherent assumption that HDR video would be preferable by the end-user over LDR video due to the more immersive and realistic visual experience provided by HDR. This assumption has led to a considerable body of research into efficient capture, processing, storage and display of HDR video. Although this is beneficial for scientific research and industrial purposes, very little research has been conducted to test the veracity of this assumption. In this paper, we conduct two subjective studies by means of a ranking and a rating-based experiment where 60 participants in total, 30 in each experiment, were tasked to rank and rate several reference HDR video scenes along with three mapped LDR versions of each scene on an HDR display, in order of their viewing preference. Results suggest that given the option, end-users prefer the HDR representation of the scene over its LDR counterpart.
\end{abstract}

Keywords HDR video · Subjective experiment · Ranking · Rating

Ratnajit Mukherjee

ratnajitmukherjee@gmail.com

1 WMG, University of Warwick, Coventry, UK

2 Jaguar Land Rover Automotive PLC, Coventry, UK

\section{Introduction}

High dynamic range (HDR) video is able to capture, process, store and display the full range of real-world lighting conditions visible to the human visual system (HVS) [2]. This, when compared to a fraction of the dynamic range displayed by existing low dynamic range (LDR) video promises to provide a more immersive and realistic viewing experience. Based on this assumption, a large body of research has been conducted to process and deliver HDR data by means of tone-mapping and compression techniques. Although this assumption is true for most of the scientific and industrial applications since HDR data provide higher precision than existing 8-bit LDR data, very little has been done to test the veracity of this assumption from an end-users' perspective.

In this paper, we investigate whether HDR video is preferred over LDR video, purely from a viewer's perspective. To that end, we selected six HDR scenes, used three separate HDR to LDR mapping techniques such that each represents a different class of mapping technique, in order to create three LDR versions of each scene. The resultant videos were displayed on an HDR screen where the reference HDR representation is absolute luminance graded from $10^{-4}$ to 4000 $\mathrm{cd} / \mathrm{m}^{2}$ and the corresponding LDR versions are graded from $10^{-4}$ to $350 \mathrm{~cd} / \mathrm{m}^{2}$. This is done to simulate the display capabilities of the HDR display and typical high-end LDR displays, respectively. Subsequently, we conducted two subjective studies by means of a ranking- and a rating-based experiment, to verify the viewing preference of end-users.

The following are the primary contributions of this work:

1. An indication by means of psychophyics experiments that HDR is significantly preferred from mapping methods. 
2. Results indicate that the ranking and rating experiments provide similar outcomes which exhibits the preference of HDR over the LDR versions.

\section{Related work}

During the past decade or so, a considerably large body of research has been conducted on tone-mapping techniques to map static HDR images and video sequences to their corresponding LDR versions in order to store and display them using legacy image/video infrastructure. The tone-mapping operators (TMOs), proposed to date, can broadly be classified as global or local TMOs. In addition, they can also be classified as non-temporally coherent TMOs or temporally coherent TMOs suitable for video tone-mapping applications. Furthermore, the availability of a multitude of TMOs has in turn led to the considerable body of research conducted in order to evaluate the TMOs, most of which were conducted by means of subjective experiments in controlled environments using a number of evaluation techniques such as rating, ranking and pairwise comparison.

Drago et al. [6] was one of the first to conduct a subjective evaluation of TMOs wherein four different HDR scenes were tone-mapped using seven different TMOs and were evaluated by 11 participants by means of a pairwise comparison technique without the HDR reference. Ledda et al. [17] conducted the first TMO evaluation using an HDR reference. 18 participants evaluated six different TMOs applied to 23 images using pair of LDR displays along with the HDR display. Several other TMO evaluations along similar lines have been conducted such as the ones conducted by Kuang et al. [16], Čadik et al. [3], etc. More recently, Eilertsen et al. [9] conducted a subjective evaluation where several temporally coherent TMOs were evaluated by means of a pairwise comparison technique. Results demonstrated that several TMOs introduced video artefacts such as flickering, ghosting and redundant saturation. Furthermore, it suggests that relatively less complex global TMOs can outperform complex local TMOs for video application. The work is of particular interest to us since it evaluates several TMOs for video applications out which one of the temporally coherent TMOs, proposed by Mantiuk et al. [18] has been used in this work. Furthermore, several other TMO evaluations have been conducted by Narwaria et al. [21], Urbano et al. [26] and Melo et al. [19]. However, it is to be noted that the above mentioned TMO evaluations were conducted with the basic assumption that although static HDR images or HDR video sequences are preferred over a tone-mapped LDR version, they are not compatible with legacy infrastructure. Therefore, the alternative is to evaluate a plethora of TMOs to identify which TMOs are capable of maximal scene reproduction.
The veracity of such an assumption was first tested by Akyüz et al. [1] where the authors conducted a series of subjective experiments to determine the best technique to display LDR images on state-of-the-art HDR displays and to identify which stages of the HDR pipeline are perceptually most critical. The first experiment conducted as a part of this study used ten different static HDR images and generated several LDR versions of each. The HDR image was subsequently displayed on a Brightside DR-37-P [25] HDR display with a peak luminance value of $3000 \mathrm{~cd} / \mathrm{m}^{2}$ and the LDR images were displayed on a commercially available Dell UltraSharp 2007FP. Results suggest that although the basic assumption that the HDR image representation would be preferred over LDR holds, it might not necessarily be the case since tone-mapped images have been ranked second to the original HDR representation. Furthermore, the study also determines that although tone-mapped images preserve more details and visibility in general, compared to a single exposure representation of the scene, it might lead to visual unnaturalness in the process as viewers are used to seeing over and under exposed areas in single exposure images. This might lead to a result where tone-mapped images have no statistically significant difference with that of single exposures.

Although the primary research question has been answered in this work, the authors focused on static HDR images only. Furthermore, several advanced perceptually motivated TMOs have been proposed since. Therefore, it is important to evaluate whether the findings by Akyüz et al. [1] hold for HDR video sequences given the current scenario where several perceptual TMOs are available. To the best of our knowledge, no such body of work exists for HDR video and a study to test the veracity of the basic assumption was the primary motivation of the work presented here.

\section{HDR to LDR mapping techniques}

Three HDR to LDR mapping techniques were chosen for this work such that each represents a different class of HDR to LDR mapping technique.

\subsection{Display adaptive tone mapping (mantiuk)}

Mantiuk et al. [18] proposed a TMO where the primary goal is to preserve the appearance of the original HDR scene including contrast, sharpness and colours by adjusting the image/video content with the pre-notion of the ambient illumination and capabilities of the target display. The authors show that such a TMO can be defined as a non-linear optimisation problem which can subsequently be simplified by reducing the degrees of freedom of the optimised system. The resultant was the introduction of a TMO with adjustable parameters that employs a piecewise linear tone-curve to map 
the HDR luminance to its corresponding LDR luminance. Given a particular display device's characteristics, the TMO produces the least distorted image in terms of visible contrast distortions which when weighed by an HVS model accounts for luminance masking, spatial contrast sensitivity and contrast masking.

The mapping technique employs image enhancement techniques to enhance the contrast of the reference image by $15 \%$. It uses a display model to account for the limitations of the target display and an HVS model based on Daly's contrast sensitivity function (CSF) [4] to derive a piecewise tone-curve which maps the reference HDR luminance to a just noticeable difference (JND) space such that the visible distortions due to the luminance mapping are minimised. Furthermore, the authors use the techniques introduced by Schlick [23] to preserve the chroma information. The proposed TMO also accounts for temporal coherence when tone mapping an HDR video sequence. Further details are available in [18].

The primary reason for choosing this HDR to LDR mapping technique is because it endeavours to reproduce the reference HDR scene with minimal visible distortion and also accounts for temporal coherence (for HDR video sequences), ambient lighting and target display. In our case the target display was set to lcd-bright to exploit the capabilities of the SIM2 HDR display. Also, this TMO in particular performs very well in comparison tests amongst other operators [19].

\subsection{Optimal exposure (optimal)}

Debattista et al. [5] proposed a two-stream based backward compatible HDR video compression algorithm which uses an exposure extraction technique [13] to create an 8-bit base stream. However, this exposure extraction can also be used as an alternative to tone-mapping HDR images or video frames wherein a single exposure extracts the maximum possible luminance information from the original HDR data that be can fitted within the allowable bit-depth of legacy imaging infrastructure which is typically $8 \mathrm{bits} / \mathrm{pixel} / \mathrm{channel}$.

The luminance information of the HDR data is calculated using the REC. 709 primaries along with the dynamic range of the image/video frame. The number of bins required to construct the histogram of the LDR image is then calculated using Freedman-Diaconis rule [12]. Subsequently, the largest contiguous area of the histogram is then chosen to fit within the required bit depth. This suffices to identify the optimal exposure which endeavours to preserve the maximum luminance information that can be extracted from the HDR image to create an LDR image.

For HDR video applications, the exposure extraction takes place in dual-loop fashion where the optimal exposure per frame is calculated into an array and the filtered information is used to extract the data from HDR frames on the second loop to create the optimally extracted LDR frames. This, in turn, helps to preserve temporal coherence across successive frames of the video. Further details are available in [5].

The primary motivation behind selecting this mapping technique is that it provides an alternative technique to extract the HDR luminance range into a single optimally calculated exposure and maps the exposure into an 8 bit LDR range analogous to an optimally metered $8 \mathrm{bit} / \mathrm{pixel} / \mathrm{channel}$ image from a camera under varying lighting conditions.

\subsection{ICAM06: image appearance model (iCAM)}

Kuang et al. [15] proposed a new image appearance model, designated iCAM06, designed specifically for HDR image rendering. Based on the $\mathrm{ICAM}$ framework [20], the new model incorporates the spatial processing models in the HVS for contrast enhancement and photo-receptor light adaptation functions that enhance local details in highlights and shadows.

The original HDR image is first converted to the CIE$\mathrm{XYZ}$ colour space and subsequently decomposed into a base and a detail layer wherein the base layer is obtained using an edge-preserving bilateral filter [7] and the detail layer is obtained by subtracting the base layer from the original image.

The base layer first undergoes chromatic adaptation which is achieved by converting the CIE-XYZ image to a spectrally sharpened RGB image using the $\mathrm{M}_{\mathrm{CAT} 02}$ transformation matrix [20]. The incomplete adaptation factor is computed as a function of adaptation luminance and the surround factor. Subsequently, the spectrally sharpened RGB image is converted from the CAT02 space to the Hunt-PointerEstevez fundamentals which is where the resultant RGB signal undergoes a non-linear tone compression using a nonlinear response function for both rods and cones derived from the Hunt Model [11]. The tone-compressed RGB image is then converted to the perceptually uniform colour opponent space IPT [8], which is desired for image attribute adjustments without affecting other attributes. To preserve the naturalness of the rendered tone-compressed image, the detail layer is enhanced to predict the Stevens effect and the P\&T channels of the base layer is enhanced to predict the Hunt effect [11]. Finally, the enhanced base and detail layers are combined to produce an enhanced perceptually uniform output image. This is displayed on the target device by converting the IPT image to an RGB signal followed by an inverse chromatic adaptation.

The primary reason for choosing this tone compression algorithm is that it provides an HVS based alternative technique to the multitude of available TMOs and yet at the same time predicts and preserves the colourfulness of the original scene. 

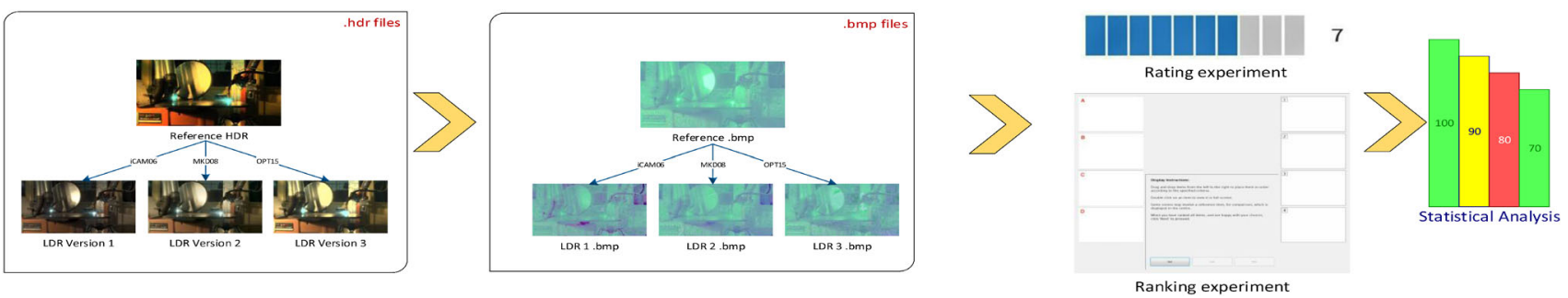

Fig. 1 An schematic diagram of the overall work flow

\section{Overview of the psychophysical studies}

In this section, we provide a brief overview of the work flow required to prepare the materials common to both the ranking and rating based experiments. A visual description of the overall work flow is given in Fig. 1. Also, a brief description of the scenes used for both the experiments is given in Table 1 .

\subsection{Motivation}

The three most commonly used techniques to conduct a subjective study are by means of ranking, rating and pairwise comparison. In this work, we have conducted two such studies by means of a ranking and a rating based experiment. The primary motivations behind choosing the techniques are (a) to investigate whether the assumed preference of HDR over LDR holds when using two different experiment designs (for additional verification) with mutually exclusive groups of participants and (b) both rating and ranking can be relatively straightforward to conduct and are of a shorter duration than a full pairwise comparison.

\subsection{Preparation of materials}

We outline the common procedure involved to prepare the HDR video content used for both the experiments. Six HDR video scenes (HDRVs), comprising of 150 frames each, were chosen such that the selected HDRVs represent a wide variety of production techniques and a large variation in dynamic range. All scenes used in this work have a resolution of $1920 \times 1080$ and were graded (in absolute luminance terms) such that the pixel values are in the range of $10^{-4}$ to 4000 $\mathrm{cd} / \mathrm{m}^{2}$. Subsequently, using the HDR to LDR mapping techniques described earlier, three corresponding LDR video

Table 1 Overview of the scenes used for the ranking- and rating-based psychophysical experiment

\begin{tabular}{|c|c|c|c|c|c|}
\hline Thumbnail & Name & Resolution & $\begin{array}{l}\text { Dynamic } \\
\text { range (stops) }\end{array}$ & Production technique & Description \\
\hline & Fireplace & $1920 \times 1080$ & 21.23 & Arri Alexa & $\begin{array}{l}\text { An outdoor winter-night scene with a } \\
\text { bright bonfire in the foreground. Scene } \\
\text { post-processed }\end{array}$ \\
\hline & Welding & $1920 \times 1080$ & 20.54 & Spheron VR & $\begin{array}{l}\text { An indoor scene of a gas welding machine } \\
\text { producing intermittent sparks of very } \\
\text { high luminance }\end{array}$ \\
\hline & CGRoom & $1920 \times 1080$ & 19.29 & Rendered & $\begin{array}{l}\text { An artificially rendered scene of the dark } \\
\text { basement with an overhead lamp } \\
\text { swinging as barrels fall from an } \\
\text { overhead shelf }\end{array}$ \\
\hline & Jaguar & $1920 \times 1080$ & 25.30 & Canon EOS 1Ds Mark II & $\begin{array}{l}\text { An side profile indoor shot of a Jaguar } \\
\text { E-Type. Bright lights are placed in the } \\
\text { room for artificially expanding the scene } \\
\text { dynamic range }\end{array}$ \\
\hline & Seine & $1920 \times 1080$ & 20.54 & Arri Alexa & $\begin{array}{l}\text { Night outdoor scene of the river Seine in } \\
\text { Paris with a brightly lit ferry producing } \\
\text { the high-luminance region of the scene. } \\
\text { Scene post-processed }\end{array}$ \\
\hline & Tears of steel & $1920 \times 1080$ & 20.35 & N.A. & $\begin{array}{l}\text { A clip extracted from the short film } \\
\text { produced as a part of the Open Movie } \\
\text { project by Blender Foundation }\end{array}$ \\
\hline
\end{tabular}


sequences (LDRVs) were created for each of six scenes. The output HDRVs and LDRVs (6 HDRVs +18 LDRVs $=24$ in total) produced were in $h d r$ format and in linear RGB colour space. This was necessary since both the HDRVs and LDRVs were subsequently converted to a SIM2 [24] HDR display suitable mode.

Since both the HDRVs and LDRVs were being displayed on the HDR display, the luminance rating of the HDRV and LDRV frames was verified using the SpectroDuo PR-680 photo-spectrometer [22], and it was ensured that the maximum luminance rating of the HDRVs was within $4000 \mathrm{~cd} / \mathrm{m}^{2}$ while the luminance rating of the LDRVs was within 350 $\mathrm{cd} / \mathrm{m}^{2}$.

\subsection{Footage}

Table 1 provides a brief description of each scene along with a tone-mapped frame, overall dynamic range and production technique.

\subsection{Materials}

Software resources used for both the ranking- and the rating-based experiment included the 24 video sequences. Hardware resources included a 47" SIM2 HDR display with a $1920 \times 1080$ native resolution, a peak luminance of $4000 \mathrm{~cd} / \mathrm{m}^{2}$ and a contrast ratio of $>10^{6}: 1$ [24]. The LDR display used in the experiments was an Alienware 23" IPS display, also with a $1920 \times 1080$ resolution, a peak luminance of $350 \mathrm{~cd} / \mathrm{m}^{2}$ and a maximum contrast ratio of $8 \times 10^{5}: 1$.

\section{Experiment 1: ranking}

In this section, we provide a brief overview of the rankingbased subjective experiment which includes a brief discussion about the design of the experiment, materials used, environment of experiment set-up, participant recruitment and the procedure followed to conduct the experiment.

\subsection{Design}

The motivation of this experiment was to rank and identify the order of viewing preference of each version (HDR/LDR), across the selected scenes. Based on their judgment of the displayed video quality (overall contrast, brightness, clarity and sharpness), the participants were tasked to rank four versions, which included the hidden reference, for each of the selected scenes, one at a time. For each sequence they had to view HDRVs/LDRVs at least once. The sequences per scene belonging to each of four versions were randomly presented in order to avoid bias. While ranking the scenes,

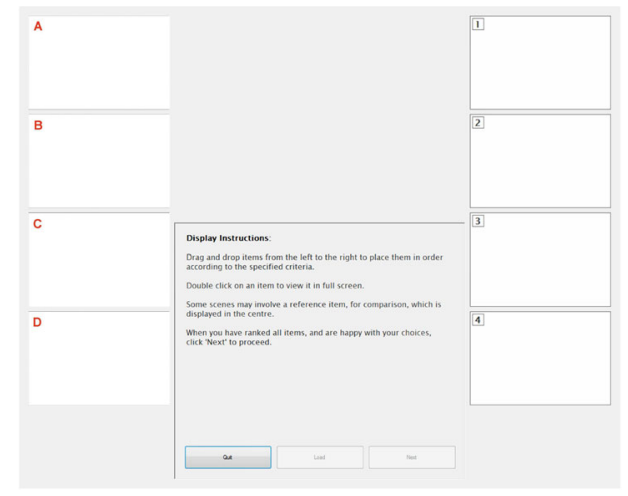

Fig. 2 Custom GUI used for the ranking experiment

participants were allowed to view the sequences as many times as required.

The independent variables in this experiment were the selected scenes and the four versions of each scene. The dependent variable in this experiment were the ranks assigned to the four versions for the selected scenes. A withinparticipants design was employed such that every participant viewed all the scenes.

\subsection{Materials}

For the purpose of the ranking experiment only five scenes were used as one scene was reserved as a demo scene, results of which would further be discarded from the final ranking results. Also, a custom graphical user interface (GUI), as shown in Fig. 2, was specifically built such that it presents four thumbnails each linked to either an HDRV (hidden reference) or an LDRV on the left side of the screen. Each thumbnail, when double-clicked, plays the linked HDRV/LDRV. Participants are tasked to view each of the videos and drag the corresponding thumbnail to the right side of the screen in order of their viewing preference. The instructions for carrying out the experiment are clearly described in the text box in the middle.

\subsection{Participants}

A total of 30 participants with an age range of 25-50 years and from various academic and corporate backgrounds took part in this experiment. All participants had normal or correctedto-normal vision.

\subsection{Environment}

Following ITU-R recommendations [14], the experiments were conducted in a room with minimal ambient lighting, below 25 lux, which is within the recommended luminance levels for a typical dark environment [10]. The distance 


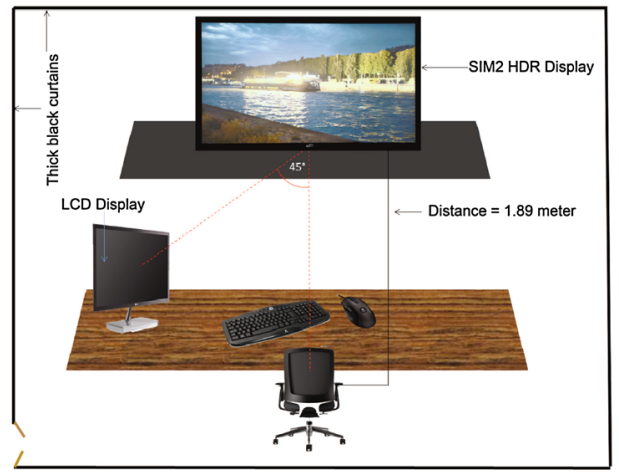

Fig. 3 Schematic diagram of the ranking experiment setup

between the HDR display and the participant was set to approximately 3.2 times the height of the HDR display; at a distance of $\approx 189 \mathrm{~cm}$ with an LCD monitor placed at an angle of $45^{\circ}$ (see Fig. 3). In order to minimise glaring, the brightness and contrast of the LCD monitor was reduced to $25 \%$.

\subsection{Procedure}

The participants were first introduced to the objectives of the experiment which was to judge the overall quality of a video footage (HDRV/LDRV). Along with the verbal introduction, the participants were given a consent form and an information leaflet. Initially, the participants were given a demonstration of the experiment using the demo sequence, the results of which were subsequently discarded from the main results. Upon completion of the demonstration, the participants were asked to proceed with ranking the remaining scenes. Based on their judgement of the displayed video quality, the participants positioned the corresponding thumbnails (labelled [A-D]) to any of the blank positions (labelled [1-4]) by means of the GUI.

\section{Experiment 2: rating}

This section provides a brief overview of the rating-based subjective experiment which includes a brief overview of the design of the experiment, materials used, experiment set up, participant recruitment and the procedure followed, respectively.

\subsection{Design}

The independent variables are the six scenes and four versions of each scene. The dependent variable in this case are the scores on a scale of [0-10] given to each of the video sequences by the participants. The participants were tasked to rate four versions for each of the selected scenes. A withinparticipants design was used and all the participants viewed
Table 2 Detailed breakup of the five groups

\begin{tabular}{ll}
\hline Group number & Number of participants \\
\hline 1 & 8 \\
2 & 6 \\
3 & 6 \\
4 & 5 \\
5 & 5 \\
\hline
\end{tabular}

all possible combinations of scenes and versions. In order to facilitate the experiment, participants were presented the stimuli in groups of five to eight participants at a time.

\subsection{Materials}

For the purpose of the rating experiment, interactive batch files were created for each group of the participants (see Sect. 6.3) such that the total 24 videos are ordered in a pseudorandom manner to be played sequentially for each group of participants. Furthermore, due to the creation of individual batch files for each group of participants, it was ensured that the ordering of videos for each batch file is also randomised.

\subsection{Participants}

A total of 30 participants, divided into five groups (see Table 2), with an age range of 20-40 years and from various academic backgrounds, took part in the rating experiment. All participants had normal or corrected-to-normal vision.

\subsection{Environment}

Unlike the ranking experiment, the rating experiment was conducted in a marginally brighter room. The ambient lighting in the room was below 50 lux, within the recommended luminance levels for a typical dark-dim environment [10]. Also, unlike the ranking experiment, where the participant controlled the ranking GUI, the conductor of the experiment controlled the interactive batch files for this experiment. Also, the LCD monitor was turned away from the participants during the experiment. A visual description of the rating environment setup is given in Fig. 4.

\subsection{Procedure}

The participants were first introduced to the objectives of the experiment and gave their consent for participating. Unlike the ranking experiment, all six HDR scenes were used in the rating experiment. The participants were tasked to rate the 24 video sequences (played individually) in order of their viewing preference on a scale of [0-10]. However, the participants 


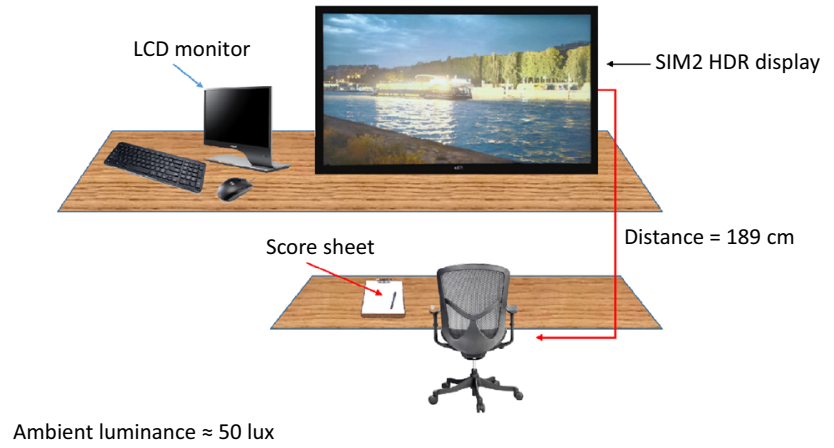

Fig. 4 Schematic diagram of the rating experiment setup

were also instructed to look for artefacts such as colour shift (common to tone-mapping techniques) and flickering (common to non-temporally coherent tone-mapping/compression techniques). The rating was performed on a hard copy score sheet which was later digitised for further analysis.

\section{Results}

In this section, we present an overview of the results obtained from the ranking- and the rating-based experiments and analyse the same.

\subsection{Ranking results}

Let the null hypothesis $H_{0}$ be that there are no significant differences between the reference HDR content and its corresponding LDR versions. The alternate hypothesis $H_{1}$ states that there are significant differences between the HDR and LDR versions. The statistical level for analysing the obtained results is assumed to be 0.05 and the sample size (total number of participants) was 30 . Furthermore, if $H_{1}$ is true, then the Kendall's coefficient of concordance $W$ (the degree of mutual agreement amongst participants) can be determined as

$W=\frac{2 \Sigma}{\left(\begin{array}{c}N \\ 2\end{array}\right)\left(\begin{array}{l}t \\ 2\end{array}\right)}-1$ where $\Sigma=\sum_{i \neq j}\left(\begin{array}{c}\alpha_{i j} \\ 2\end{array}\right)$.

The significance of $W$ can be analysed using chi-squared statistics such that

$\chi^{2}=\frac{t(t-1)(1+W(N-1))}{2}$.

$\chi^{2}$ is asymptotically distributed with $\frac{t(t-1)}{2}$ degrees of freedom, where $t=4$ represents the number of operators (HDR $+3 \mathrm{LDR}$ ), and $N=30$ represents the number of participants. A significance between scores suggests that the perceived image quality of two operators, when compared with each other, are different although no conclusions can be drawn for cases of similarity.
The data obtained from the ranking experiment need to be tested for homogeneity and any outliers must be removed before further analysis can be performed. To that extent, the data obtained from the ranking experiment are folded across all scenes to obtain a grand average. We tested for outliers by means of a histogram plot and stem-and-leaf display method. The outliers are then identified using a box-andwhisker plot and are subsequently removed from the raw data. This ensures that the grand average has normally distributed data points.

Following the above mentioned technique, three outliers were identified in the raw ranking data which were subsequently removed, thus reducing the sample size to 27 . The resultant data were further analysed using statistical non-parametric tests, such as Kendall's, of concordance for $K$-related samples.

The overall ranking scores demonstrate a significance of $p<0.05$. Therefore, $H_{0}$ is rejected and $H_{1}$ is accepted. This means that the ranking results averaged over the sample size of 27 exhibit significant differences between the four operators (versions) for each of the five scenes as well as the grand average of the five scenes. Before the result of the full pairwise comparison on the four operators (on the grand average) is presented, we present the mean ranking scores assigned to each operator per sequence as well as the derived average scores (folded across five scenes) along with their variation denoted by $95 \%$ confidence intervals in Fig. 5 .

Next, we present the result of the full pairwise comparison on the grand average data in Table 3 which demonstrates significant differences between the operators. However, operators within the same group exhibit no statistically significant differences with each other.

\subsection{Rating results}

Analogous to the process mentioned in Sect. 7.1, the combined results obtained from the rating-based subjective experiment were folded across the six scenes and the grand average was tested for outliers. Based on the box-and-whisker plot, two outliers were identified and removed from the raw data set thus reducing the sample size to 28 . Using the resultant data, we present the mean rating scores for each of the four operators per sequence and for the derived grand average in Fig. 6.

Subsequently, the null hypothesis $H_{0}$ was tested using the one-way repeated measures analysis of variance (ANOVA). The results of the ANOVA indicate a statistically significant difference between the four operators. As the resultant data fail Mauchly's sphericity test, $p<0.01$, the GreenhouseGaussier post-hoc correction was applied, $F(1.588,81)=$ 10.073, $p<0.05, \eta=0.272$, which also indicates significant difference between the four operators. Follow-up pairwise comparisons, on the grand average, indicate the 


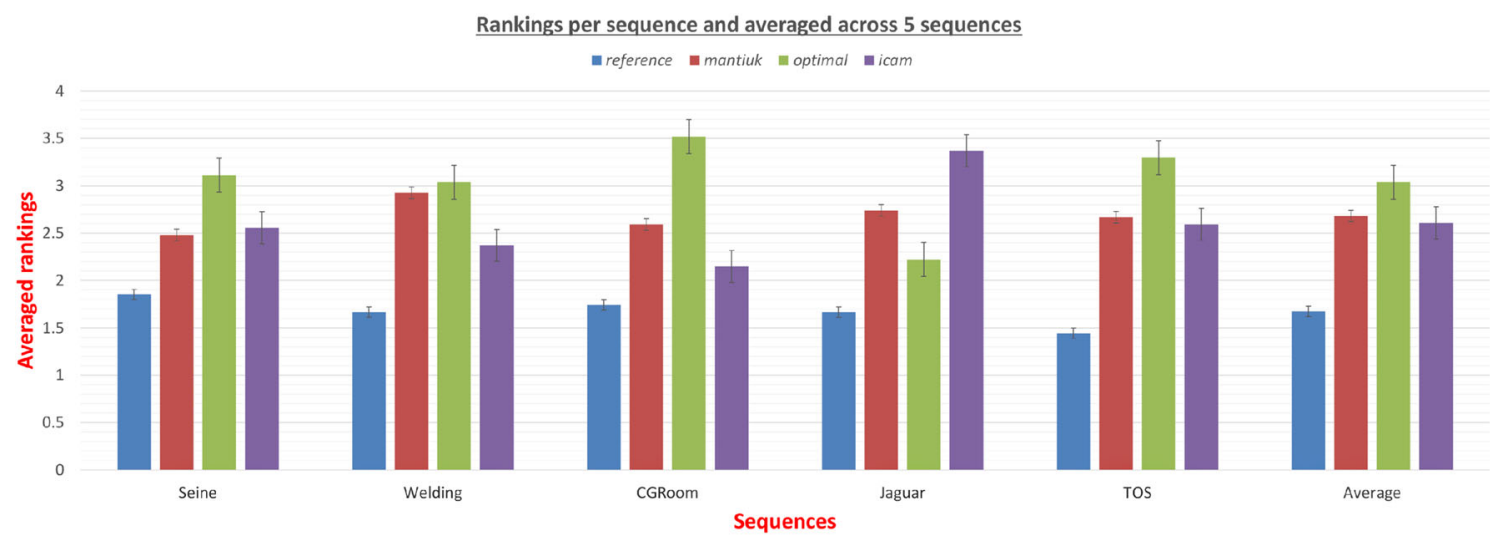

Fig. 5 Overall ranking scores-per sequence (averaged over 27 participants) and averaged ranks across five scenes (lower is better)

Table 3 Mean ranks with Kendall $W$, averaged across five scenes and 27 participants (lower is better)

Fig. 6 Overall rating scores-per sequence (averaged over 28 participants) and average scores across all six scenes and 28 participants (higher is better)

Table 4 Mean rating scores with Kendall $W$, averaged across six scenes and 28 participants (higher is better)

\begin{tabular}{|c|c|c|c|c|c|c|}
\hline Sequence & \multicolumn{4}{|c|}{ Methods } & Kendall (W) & $x^{2}$ \\
\hline $\begin{array}{l}\text { Average across } 27 \\
\text { participants and } 5\end{array}$ & $\begin{array}{c}\text { reference } \\
1.54\end{array}$ & $\begin{array}{c}\text { mantiuk } \\
2.54\end{array}$ & $\begin{array}{l}\text { icam } \\
2.59\end{array}$ & $\begin{array}{c}\text { optimal } \\
3.33\end{array}$ & 0.335 & 37.137 \\
\hline
\end{tabular}

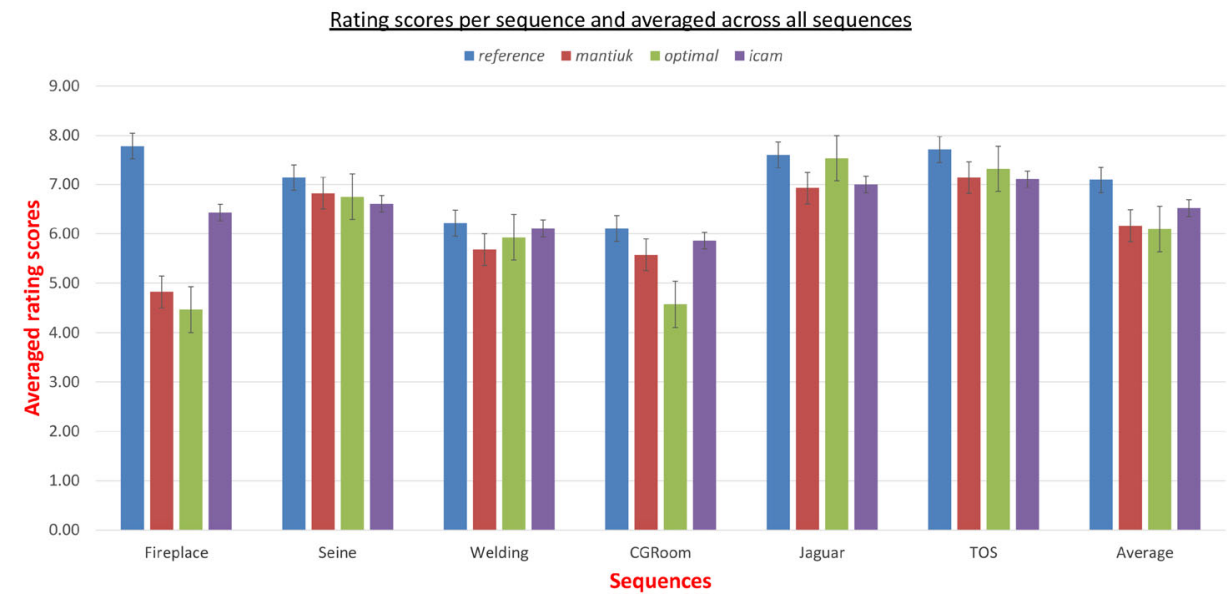

\begin{tabular}{|c|c|c|c|c|c|}
\hline Sequence & \multicolumn{4}{|c|}{ Methods } & Kendall (W) \\
\hline $\begin{array}{l}\text { Average across } 28 \\
\text { participants and } 6\end{array}$ & $\begin{array}{c}\text { reference } \\
7.10\end{array}$ & $\begin{array}{l}\text { icam } \\
6.52\end{array}$ & $\begin{array}{c}\text { mantiuk } \\
6.16\end{array}$ & $\begin{array}{c}\text { optimal } \\
6.09\end{array}$ & 0.260 \\
\hline
\end{tabular}

groups into which the operators can be assigned and the Kendall's coefficient of concordance which denotes the degree of agreement amongst the participants as shown in Table 4.

\section{Discussion}

The results from both experiments are overall fairly similar. They indicate a preference for HDR and less of a prefer- ence for the LDR mapping methods. There is a distinction between the preference of the mapping methods, however, although for the most part no significant difference between the mapping methods was encountered apart from the icam being preferred in the rating experiment.

The mean ranks with $95 \%$ confidence interval error bars for each operator as shown in Fig. 5 clearly exhibit a significant difference between the reference HDR and the three LDR versions for each of the five scenes as well as the grand average. However, the difference in between the 
LDR versions are less significant. Analysis of the ranking scores, averaged across the five scenes, also exhibits the same characteristics wherein the reference HDR videos exhibit statistically significant difference with that of the LDR versions mantiuk, icam and optimal as shown in Table 4. However, there are no statistically significant differences in between the three LDR versions. Furthermore, it is to be noted that the Kendall's coefficient of concordance for the grand average ranks, as shown in Table 3, exhibits a low concordance value which also indicates a degree of ambivalence amongst the participants.

Similarly, the mean rating scores as shown in Fig. 6 exhibit a significant difference between the reference HDR and the corresponding three LDR versions for each of the six scenes as well as the grand average. Furthermore, the results of the pairwise comparison from the repeated measures ANOVA demonstrate that the reference HDR is significantly different than icam which in itself exhibits statistically significant difference with mantiuk and optimal.

Although the results presented in this work involve HDR video sequences, they bear similarity with the findings of the previous study by Akyuz et al. [1] which used static HDR and tone-mapped images. Both studies demonstrate a statistically significant difference between the reference HDR images/videos and the corresponding tone-mapped versions of the same. Even though many advanced tone-mapping techniques have been proposed since the previous work which endeavour to replicate the overall scene contrast to a higher degree than previous TMOs, some of which has been used in this work, there is evidence that given the correct viewing conditions and properly prepared materials, HDR video supersedes LDR video. However, there are limitations in this study. Only six HDR scenes were used in this work out of which five were used for the ranking-based experiments. Results might vary if the number of scenes and HDR to LDR mapping techniques are increased. Furthermore, the viewers were presented with independent visual stimuli which are not a part of any contextual narrative upon which the results might also vary.

\section{Conclusion}

In this work, we asked a fundamental question as to whether HDR video is indeed preferred over legacy LDR video, purely from the viewers perspective. The technical advantages of HDR video over LDR video and the multitude of TMOs, some of which reproduce a more artistic representation of the original scene, were not considered in this work. Therefore, we used three HDR to LDR mapping techniques such that they are able to reproduce the original reference to the extent possible and conducted two subjective experiments with 60 participants in total, 30 in each group (mutually exclusive), both of which demonstrate that there exists a statistically significant difference between the HDR (more realistic) representation of a scene and its LDR counterparts where the former is preferred by the end-users.

Acknowledgments We would like to thank Technicolor SA for the "Seine" footage and all the participants of the experiments. This work is funded by EPSRC EP/K014056/1 with Jaguar Land Rover: "PSi Theme 7: Visualisation and Virtual Experience". Chalmers and Debattista are Royal Society Industrial Fellows, for whose support we are most grateful.

Open Access This article is distributed under the terms of the Creative Commons Attribution 4.0 International License (http://creativecomm ons.org/licenses/by/4.0/), which permits unrestricted use, distribution, and reproduction in any medium, provided you give appropriate credit to the original author(s) and the source, provide a link to the Creative Commons license, and indicate if changes were made.

\section{References}

1. Akyüz, A.O., Fleming, R., Riecke, B.E., Reinhard, E., Bülthoff, H.H.: Do hdr displays support ldr content? A psychophysical evaluation. In: ACM SIGGRAPH 2007 Papers, SIGGRAPH'07. ACM, New York (2007). doi:10.1145/1275808.1276425

2. Banterle, F., Artusi, A., Debattista, K., Chalmers, A.: Advanced High Dynamic Range Imaging: Theory and Practice. CRC Press, Boca Raton (2011)

3. Čadík, M., Wimmer, M., Neumann, L., Artusi, A.: Evaluation of HDR tone mapping methods using essential perceptual attributes. Comput. Graph. 32(3), 330-349 (2008). doi:10.1016/ j.cag.2008.04.003. http://www.sciencedirect.com/science/article/ pii/S0097849308000460

4. Daly, S.J.: Visible differences predictor: an algorithm for the assessment of image fidelity (1992). doi:10.1117/12.135952

5. Debattista, K., Bashford-Rogers, T., Selmanović, E., Mukherjee, R., Chalmers, A.: Optimal exposure compression for high dynamic range content. Vis. Comput. 31(6-8), 1089-1099 (2015)

6. Drago, F., Martens, W.L., Myszkowski, K., Seidel, H.P.: Perceptual evaluation of tone mapping operators. In: ACM SIGGRAPH 2003 Sketches and Applications, SIGGRAPH'03, pp. 1-1. ACM, New York (2003). doi:10.1145/965400.965487

7. Durand, F., Dorsey, J.: Fast bilateral filtering for the display of high-dynamic-range images. In: Proceedings of the 29th Annual Conference on Computer Graphics and Interactive Techniques, SIGGRAPH'02, pp. 257-266. ACM, New York (2002). doi:10. $1145 / 566570.566574$

8. Ebner, F., Fairchild, M.D.: Development and testing of a color space (ipt) with improved hue uniformity. Color Imaging Conf. 1998(1), 8-13 (1998). http://www.ingentaconnect.com/content/ ist/cic/1998/00001998/00000001/art00003

9. Eilertsen, G., Wanat, R., Mantiuk, R.K., Unger, J.: Evaluation of tone mapping operators for HDR-video. Comput. Graph. Forum 32(7), 275-284 (2013). doi:10.1111/cgf.12235

10. Engineering toolbox: illuminance-recommended light levels. http://www.engineeringtoolbox.com/light-level-rooms-d_708. html

11. Fairchild, M.D.: Color Appearance Models. Wiley, New York (2013)

12. Freedman, D., Diaconis, P.: On the histogram as a density estimator: L 2 theory. Probab. Theory Relat. Fields 57(4), 453-476 (1981)

13. Hirakawa, K., Wolfe, P.: Optimal exposure control for high dynamic range imaging. In: 2010 17th IEEE International Confer- 
ence on Image Processing (ICIP), pp. 3137-3140 (2010). doi:10. 1109/ICIP.2010.5654059

14. ITU: Recommendation itu-r bt.500-13: Methodology for the subjective assessment of the quality of television pictures. Tech. rep., International Telecommunication Union (2012). http://www. itu.int/dms_pubrec/itu-r/rec/bt/R-REC-BT.500-13-201201-I!! PDF-E.pdf

15. Kuang, J., Johnson, G.M., Fairchild, M.D.: icam06: a refined image appearance model for HDR image rendering. J. Vis. Commun. Image Represent. 18(5), 406-414 (2007). doi:10.1016/j. jvcir.2007.06.003. http://www.sciencedirect.com/science/article/ pii/S1047320307000533. (Special issue on High Dynamic Range Imaging)

16. Kuang, J., Yamaguchi, H., Liu, C., Johnson, G.M., Fairchild, M.D.: Evaluating hdr rendering algorithms. ACM Trans. Appl. Percept. 4(2) (2007). doi:10.1145/1265957.1265958

17. Ledda, P., Chalmers, A., Troscianko, T., Seetzen, H.: Evaluation of tone mapping operators using a high dynamic range display. In: ACM SIGGRAPH 2005 Papers, SIGGRAPH'05, pp. 640-648. ACM, New York (2005). doi:10.1145/1186822.1073242

18. Mantiuk, R., Daly, S., Kerofsky, L.: Display adaptive tone mapping. In: ACM SIGGRAPH 2008 Papers, SIGGRAPH'08, pp. 68:168:10. ACM, New York (2008). doi:10.1145/1399504.1360667

19. Melo, M., Bessa, M., Debattista, K., Chalmers, A.: Evaluation of tone-mapping operators for HDR video under different ambient luminance levels. Comput. Graph. Forum 34(8), 38-49 (2015). doi:10.1111/cgf.12606

20. Moroney, N., Fairchild, M.D., Hunt, R.W., Li, C., Luo, M.R., Newman, T.: The ciecam02 color appearance model. In: Color and Imaging Conference, vol. 2002, pp. 23-27. Society for Imaging Science and Technology (2002)

21. Narwaria, M., Da Silva, M., Le Callet, P., Pepion, R.: Single exposure vs tone mapped high dynamic range images: a study based on quality of experience. In: 2014 Proceedings of the 22nd European Signal Processing Conference (EUSIPCO), pp. 2140-2144 (2014)

22. Photo Research Inc.: PR-655, PR-670, PR-680 and PR-680L SpectraScan Spectroradiometers. http://www.photoresearch.com/sites/ default/files/PR-6\%20Series\%20\%20Brochure_1.pdf

23. Schlick, C.: Quantization techniques for visualization of high dynamic range pictures. In: Photorealistic Rendering Techniques, pp. 7-20. Springer, New York (1995)

24. SIM2 Multimedia: SIM2 HDR47. http://www.sim2hdr.com/ hdrdisplay/hdr47e_s_4k

25. Technologies, B.: Brightside dr-37-p. http://www.bit-tech.net/ hardware/2005/10/04/brightside hdr_edr/8

26. Urbano, C., Magalhes, L., Moura, J., Bessa, M., Marcos, A., Chalmers, A.: Tone mapping operators on small screen devices: an evaluation study. Comput. Graph. Forum 29(8), 2469-2478 (2010). doi:10.1111/j.1467-8659.2010.01758.x

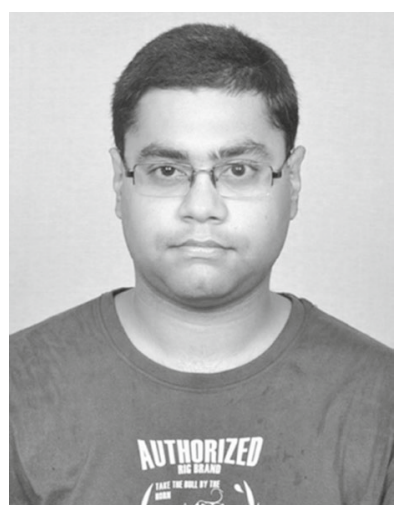

Ratnajit Mukherjee is a Ph.D. student in Visualisation Group at the University of Warwick. His research interests include HDR, video compression, signal and image processing. He completed his Bachelor of Technology degree, with honours in Information Technology from West Bengal University of Technology, WB, India, and conducted research and development of Embedded Assistive Technology devices at the Indian Institute of Technology, Kharagpur, India.

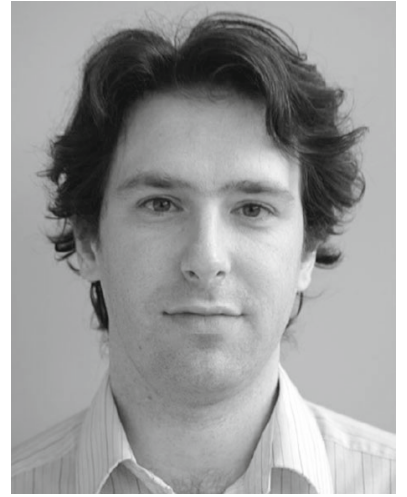

Kurt Debattista is an Associate Professor at the University of Warwick. He has a Ph.D. from the University of Bristol, an M.Sc. in Psychology, an M.Sc. in Computer Science and a B.Sc. in Mathematics and Computer Science. His research interests include HDR imaging, high-fidelity computer graphics, serious games and parallel computing.

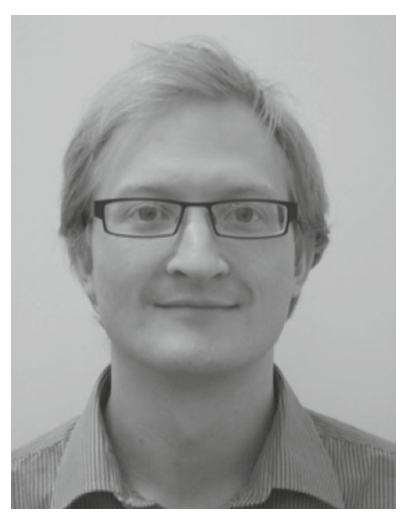

Thomas Bashford-Rogers has a degree in Computer Science from the University of Bristol and a doctorate in Computer Graphics from the University of Warwick. His research interests include global illumination, raytracing and Monte Carlo methods. He is currently a Research Fellow at the University of Warwick.

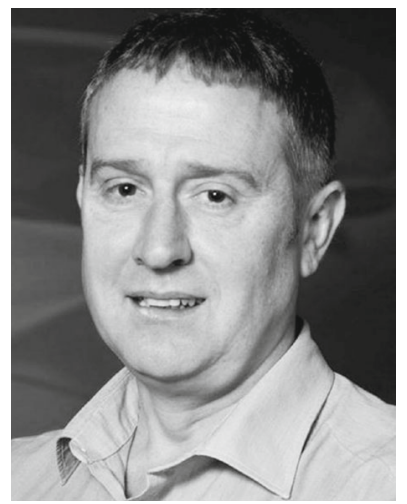

Brian Waterfield is the Virtual Reality and High-end Visualisation Technical Lead at Jaguar Land Rover. He has an M.Sc. with distinction from Salford University in Virtual Reality and Gaming Technology, and has headed up the introduction of virtual reality into JLR. In 2008 he developed the VRCentre that has evolved into the Virtual Innovation Centre at JLR, he is now working at the very forefront of research into sensory immersion both in process and product within the Vehicle design and development process.

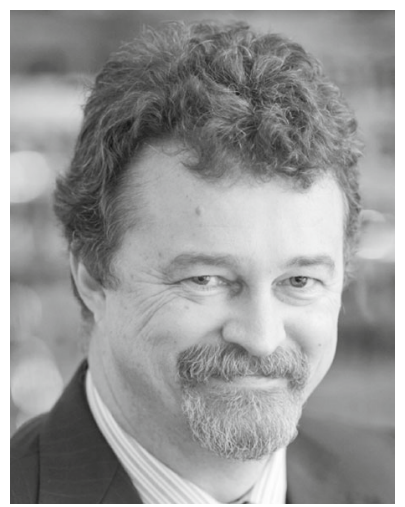

Alan Chalmers is Professor of Visualisation at the University of Warwick. He has a Ph.D. in Computer Science from the University of Bristol, 1991 and an M.Sc. with distinction from Rhodes University, SA, 1984. He has published over 210 papers in journals and international conferences on highfidelity virtual environments, multi-sensory perception, and HDR imaging. 